\title{
Modelling the optics of high resolution liquid crystal devices by the finite differences in the frequency domain method
}

\author{
Mengyang Yang, Sally E. Day and F. Aníbal Fernández \\ Department of Electronic and Electrical Engineering \\ University College London, \\ London WC1E 7JE, UK \\ a.fernandez@ucl.ac.uk
}

\begin{abstract}
A procedure combining accurate liquid crystal and electromagnetic modelling is developed for the analysis of wave propagation through liquid crystal devices. This is required to study the optics of high resolution liquid crystal cells or cells containing very small features, where diffraction effects occur. It is also necessary for the study of optical waveguiding devices using liquid crystal as variable permittivity substrates. An accurate finite element modelling program is used to find the permittivity tensor distribution, which is then used to find the response of the device to an excitation electromagnetic field by means of a finite difference in the frequency domain (FDFD) approach.
\end{abstract}

Keywords- Liquid crystal modelling; Finite differences method; Wave propagation in anisotropic inhomogeneous media; Liquid crystal optics

\section{INTRODUCTION}

This work concerns the development of a computational method to analyse electromagnetic wave propagation through liquid crystal devices. Methods used to study the optics of liquid crystal cells for traditional displays are not suitable for high resolution devices where liquid crystal defects or disclinations are important, when the cell contains small features or in general, when diffraction effects occur and cannot be ignored or are important in the device operation. The same method is also necessary to study the propagation of electromagnetic waves in tuneable or reconfigurable optical devices that use liquid crystal substrates as a variable permittivity material. Liquid crystals are anisotropic liquids where their internal molecular orientation depends of elastic and electric forces, resulting in a point-by-point inhomogeneous and anisotropic permittivity.

The procedure consists of two stages. In the first, a finite element method is used to find the permittivity tensor distribution in the liquid crystal by performing an accurate liquid crystal modelling of its orientation distribution [1], [2]. The permittivity distribution is then mapped on a finite difference grid where an FDFD method is constructed to solve the problem globally, (not as a stepping procedure) starting from the discretisation of Maxwell's equations and using the total-field/scattered-field approach [3], [4].

\section{LIQUID CRYSTAL AND ELECTROMAGNETIC WAVE PROPAGATION MODELLING}

\section{A. Liquid crystal modelling}

The liquid crystal modelling used in this work consists of a variational approach to the Landau-de Gennes theory implemented with the formalism of Qian and Sheng [1], [5]. This is a continuum theory in which the liquid crystal orientation is defined by the order tensor Q that not only describes the local orientation but also takes into account order variations. This is adequate for the accurate modelling of liquid crystal structures containing defects or other regions of order parameter variation. The permittivity distribution is then found as $\overline{\bar{\varepsilon}}=\varepsilon_{\perp} \overline{\overline{\mathbf{I}}}+\Delta \varepsilon \overline{\overline{\mathbf{n n}}}$ where $\hat{n}$ is the liquid crystal director, $\varepsilon_{\perp}$ is the relative permittivity calculated in the direction perpendicular to the long axis of the liquid crystal molecules and $\Delta \varepsilon$ is the dielectric anisotropy.

Solution is sought as the stationary states of the free energy functional [1]:

$$
\mathcal{F}(\mathbf{Q}, \varphi)=\int_{\Omega}\left\{f_{D}(\mathbf{Q})+f_{B}(\mathbf{Q})-f_{E}(\mathbf{Q}, \varphi)\right\} d \Omega+\int_{\Gamma} f_{S}(\mathbf{Q}) d \Gamma
$$

Here $f_{\mathrm{B}}$ is the bulk or thermotropic energy density, which can be expressed as a fourth order expansion:

$$
f_{B}(\mathbf{Q})=\frac{1}{2} A \operatorname{tr}\left(\mathbf{Q}^{2}\right)+\frac{1}{3} B \operatorname{tr}\left(\mathbf{Q}^{3}\right)+\frac{1}{4} C \operatorname{tr}\left(\mathbf{Q}^{4}\right)
$$

where $A, B$ and $C$ are the material bulk constants. The elastic distortion energy $f_{\mathrm{D}}$ can be expressed as:

$$
f_{D}(\mathbf{Q})=\frac{1}{2}\left\{L_{1} \mathbf{Q}_{\alpha \beta, \gamma} \mathbf{Q}_{\alpha \beta, \gamma}+L_{2} \mathbf{Q}_{\alpha \beta, \beta} \mathbf{Q}_{\alpha \gamma, \gamma}+L_{6} \mathbf{Q}_{\alpha \beta} \mathbf{Q}_{\gamma \lambda, \alpha} \mathbf{Q}_{\gamma \lambda, \beta}\right\}(3)
$$

and the $L_{i}$ terms represent elastic parameters. The term $f_{\mathrm{S}}$ represents the surface anchoring energy density at the bounding surfaces of the LC region [2]. Finally, reorientation of the LC 
is induced by the application of an external electric field. This is accounted for via the electric energy density, $f_{\mathrm{E}}$ expressed as:

$$
f_{E}(\mathbf{Q}, \varphi)=\frac{1}{2} \varepsilon_{0}(\nabla \varphi \cdot \overline{\bar{\varepsilon}} \cdot \nabla \varphi)-\vec{P} \cdot \nabla \varphi
$$

Here, $\varphi$ is the electric potential and $P$ is the flexoelectric polarisation.

The solution to (1) is found by solving the dynamic dissipation equation

$$
\frac{\partial \mathcal{F}(Q, \varphi)}{\partial Q_{i j}}+\frac{\partial D}{\partial \dot{Q}_{i j}}=0
$$

where the dot denotes time-derivative and the dissipation function $D$ depends on Q, $\varphi$ and the velocity field $v$ which is found solving the incompressible Navier Stokes equation using a Galerkin approach [1]. In this work, the resulting equation is solved using first-order three-dimensional tetrahedral elements allowing accurate modeling of LC structures with complex geometries.

\section{B. Electromagnetic modelling}

The calculation method starts by formulating Maxwell's curl equations in the form:

$$
\nabla \times \vec{E}=k_{0} \tilde{\mu}_{r} \overrightarrow{\tilde{H}} \quad \nabla \times \overrightarrow{\tilde{H}}=k_{0} \tilde{\varepsilon}_{r} \vec{E}
$$

where $\overrightarrow{\tilde{H}}=-j \eta_{0} \vec{H}$ is the scaled magnetic field. Using the total-field/scattered-field approach [3], [4], stretched coordinates perfectly matched layers (sc-PML), periodic conditions and/or electric walls are used to limit the computational domain. The sc-PML tensor is defined as:

$$
S=\left[\begin{array}{ccc}
1 / s_{x} & 0 & 0 \\
0 & 1 / s_{y} & 0 \\
0 & 0 & 1 / s_{z}
\end{array}\right]
$$

where the $s_{\xi}, \xi=x, y, z$ are the coordinate stretching functions which in here are chosen as in [7]. These are incorporated into the equations as the modified material tensors $\tilde{\mu}_{r}$ and $\tilde{\varepsilon}_{r}$ in (5) which become:

$$
\tilde{\varepsilon}_{r}=S \varepsilon_{r} S / \operatorname{det}(S), \quad \tilde{\mu}_{r}=S \mu_{r} S / \operatorname{det}(S)
$$

The equations in (5) are first discretized using a Yee grid and from the discrete equations the magnetic field is eliminated resulting in a matrix problem involving only the electric field components [4] defined on a simple, non-staggered grid. It is convenient assembling the matrix so the solution vector is ordered by points on the grid rather than by field component, so the values corresponding to the same point are consecutive. This results in a block tridiagonal matrix with a narrower bandwidth than what would result from a different ordering. It also facilitates the use of a preconditioning technique like those described in [7] and [8].

The solution of this problem normally consists of oscillatory functions involving many full cycles in all directions over the computational domain, requiring a large number of mesh points. Furthermore, the matrix problem is indefinite and ill-conditioned and needs the careful selection of a preconditioning method is needed. See for example [7].

\section{RESULTS}

As example of application of this method, a cylindrical liquid crystal microlens is modelled next. This consists of a glass cylindrical microlens of dimensions $200 \times 500 \mu \mathrm{m}$ placed on the bottom surface of a liquid crystal cell of $280 \times 75 \mu \mathrm{m}$ with air above. The calculation window is $280 \times 150 \mu \mathrm{m}$. Fig. 1 shows the electric field distribution over the complete structure ( $E_{y}$ component, perpendicular to the cross-section shown).

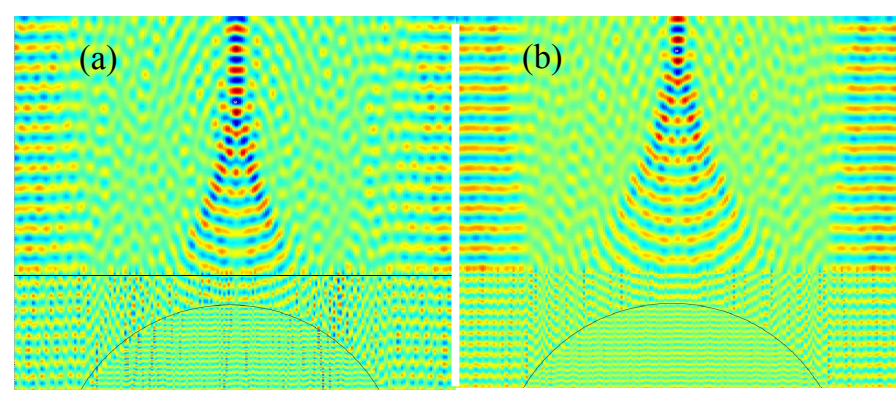

Fig. 1 (a) Shows the $E_{y}$ field distribution when the liquid crystal is unswitched (no voltage applied) and (b), when the liquid crystal is fully switched.

The effect of the liquid crystal switching is to reduce the optical refractive index in the direction of the electric field $(y)$, thus increasing the focal length, as shown in Fig. 1.

The second example corresponds to a small liquid crystal cell of $10 \times 5 \mu \mathrm{m}$ where a defect appears when an appropriate voltage is applied between the electrodes, see Fig. 2.

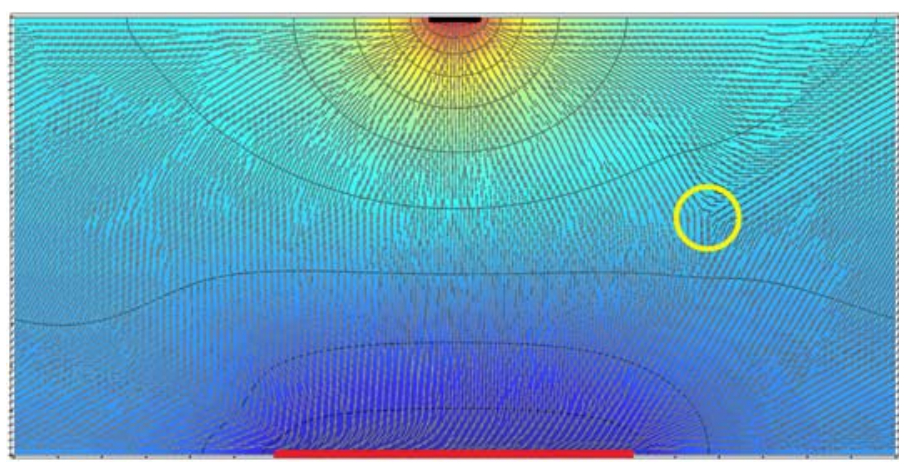

Fig. 2 Director distribution over a liquid crystal cell showing a defect (marked with yellow circle) when a selected voltage is applied to electrodes shown here with the red and the black bars.

The effect of the presence of the defect is to introduce an abrupt phase variation of the optical field in the top surface when the cell is illuminated by a plane wave from the bottom. The phase distribution over the top surface is shown in Fig. 3 
where it is compared with a fitted ideal distribution for an element in a blazed grating.

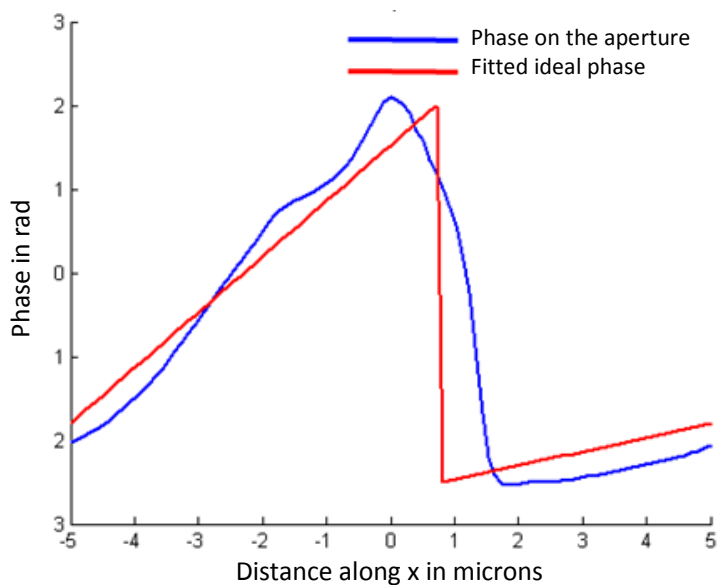

Fig. 3 Phase of the optical field in the top surface of the liquid crystal cell (shown in blue) compared with an ideal distribution for a phase grating (in red)

The corresponding far-field diffraction patterns of arrays of 10 cells are shown in Fig. 4.

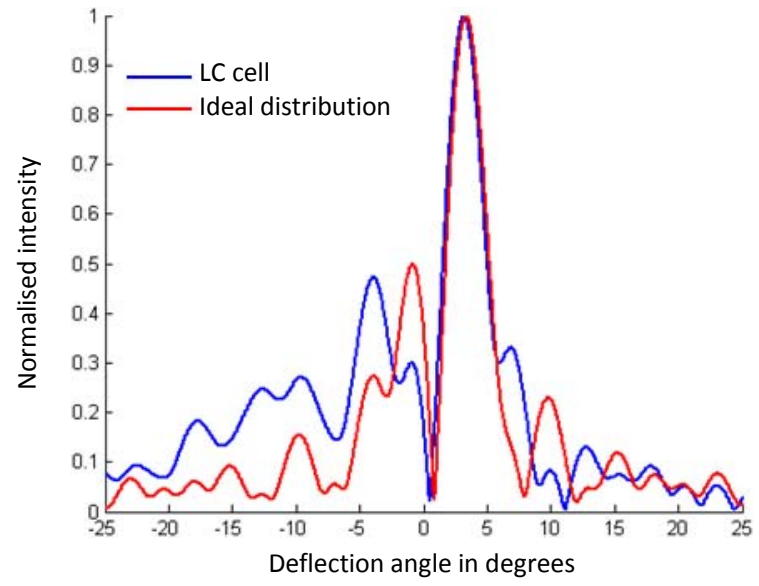

Fig. 4 Far field diffraction pattern of an array of 10 cells, each with the phase distribution shown in Fig. 3.

\section{CONCLUSIONS}

A procedure that combines accurate liquid crystal modelling with a full field analysis of the electromagnetic field that propagates through a liquid crystal cell has been shown and demonstrated with examples. The liquid crystal modelling is used to find the permittivity distribution over the liquid crystal region and is capable to model accurately liquid crystal order variations and defects. The electromagnetic modelling leads to the solution of a single, sparse matrix equation of order $3 \mathrm{~N}$, where $\mathrm{N}$ is the total number of points in a finite differences grid and needs careful selection of a preconditioner for an efficient iterative solution.

\section{REFERENCES}

[1] R. James, E. Willman, F.A. Fernandez and S.E. Day, "Finite-element modeling of liquid-crystal hydrodynamics with a variable degree of order," IEEE Trans. Electron Devices, vol. 53, No 7, pp. 1575-1582, July 2006.

[2] E. Willman, F.A. Fernandez, R. James and S.E. Day, "Modeling of Weak Anisotropic Anchoring of Nematic Liquid Crystals in the Landaude Gennes Theory", IEEE Trans Electron Devices, vol. 54, No 10, pp. 2630-2637, Oct. 2007.

[3] K. Umashankar and A. Taflove, "A novel method to analyse electromagnetic scattering of complex objects," IEEE Trans. Electromagn. Compat., vol 24, No 4, pp. 397-405, Nov. 1982.

[4] R.C. Rumpf, "Simple implementation of arbitrary shaped totalfield/scattered-field regions in FDFD," Progress in Electromagnetic Research B, vol. 36, pp. 221-248, 2012.

[5] T. Qian and P. Sheng, "Generalized hydrodynamic equations for nematic liquid crystals," Phys. Rev. E, Stat. Phys. Plasmas Fluids Relat. Interdiscip. Top., vol. 58, Nº 6, pp. 7475-7485, Dec. 1998.

[6] W.C. Chew and W.H. Weedon, A 3_d perfectly matched medium from modified Maxwell's equations with stretched coordinates," Microwave Optical Technol. Lett., vol. 7, No 13, pp. 599-604, Sept. 1994.

[7] P. Tsuji and L. Ying, "A sweeping preconditioner for Yee's finite difference approximation of time-harmonic Maxwell's equations," Front. Math. China, vol. 7, No 2, pp.347-363, 2011.

[8] B. Engquist and L. Ying, "Sweeping preconditioner for the Helmholtz equation: moving perfectly matched layers," Multiscale Model Simul, vol. 9, pp.686-710, 2011. 